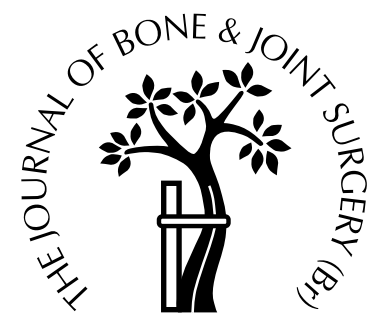

\title{
The role of angiography in the management of haemorrhage from major fractures of the pelvis
}

\author{
R. E. Cook, J. F. Keating, I. Gillespie \\ From the Royal Infirmary of Edinburgh, Scotland
}

$I^{\mathrm{n}}$ a series of 150 consecutive patients with unstable fractures of the pelvis, angiography was performed in $23 \mathbf{( 1 5 \% )}$ who had uncontrolled hypotension. There were three anteroposterior compression (APC), eight lateral compression (LC) and 12 vertical shear (VS) injuries. Arterial sources of haemorrhage were identified in $18(78 \%)$ patients and embolisation was performed. Angiography was required in $28 \%$ of VS injuries. The morphology of the fracture was not a reliable guide to the associated vascular injury. Ten $\mathbf{4 3 \%}$ ) patients died, of whom six had had angiography as the first therapeutic intervention. Five of these had a fracture which was associated with an increase in pelvic volume (APC or VS) which could have been stabilised by an external fixator. Based on our findings we recommend skeletal stabilisation and, if indicated, laparotomy to deal with sources of intraperitoneal blood loss before pelvic angiography. Embolisation of pelvic arterial bleeding is a worthwhile procedure in patients with hypotension which is unresponsive to these interventions.

J Bone Joint Surg [Br] 2002;84-B:178-82.

Received 3 April 2001; Accepted after revision 24 July 2001

Serious haemorrhage is a well-recognised complication of unstable pelvic fractures ${ }^{1-4}$ and is one of the principal causes of early mortality. ${ }^{5}$ Various forms of treatment have been advocated for the early management of the hypotensive patient with a fracture of the pelvis. These include the application of an external fixator, the use of the pneumatic anti-shock garment, ${ }^{7}$ and the application of specially designed pelvic clamps. ${ }^{2}$ Laparotomy and packing of

R. E. Cook, FRCS Ed, Specialist Registrar

J. F. Keating, MPhil, FRCS Ed, Consultant Orthopaedic Surgeon

Edinburgh Orthopaedic Trauma Unit

I. Gillespie, FRCR, Consultant Radiologist

Department of Radiology

Royal Infirmary of Edinburgh, Lauriston Place, Edinburgh EH3 9YY, UK.

Correspondence should be sent to Mr J. F. Keating.

(C)2002 British Editorial Society of Bone and Joint Surgery 0301-620X/02/212324\$2.00 the pelvic retroperitoneum have been suggested but are not commonly practised. ${ }^{8,9}$

Pelvic angiography and embolisation of bleeding vessels have been recommended as having both a diagnostic and therapeutic role in the management of pelvic bleeding, ${ }^{10}$ but the exact role of this technique in the management of fractures of the pelvis remains uncertain. Anatomical studies have suggested that the surfaces of the fracture and veins, rather than arteries, are the major sources of haemorrhage in these patients. ${ }^{11}$ The time taken to perform angiography may be considerable and may compromise the management, especially if other serious injuries are present.

We have carried out a critical analysis of the use of angiography and embolisation in the management of the hypotensive patient with an unstable fracture of the pelvis in order to define its role and optimal timing in relation to other forms of treatment.

\section{Patients and Methods}

Between 1988 and 2000, we treated 150 consecutive major fractures of the pelvis (Fig. 1). Details of each patient, including age, gender and the mechanism of injury were noted at the time of admission. There were 104 males and 46 females with a mean age of 40 years ( 8 to 88 ). The mechanism of injury was a motor-vehicle accident in 106 patients $(71 \%)$ of whom $43(29 \%)$ were car occupants, 44 (29\%) pedestrians, 16 (11\%) motorcyclists, and three (2\%) cyclists. A fall from a height was the cause in 41 patients (27\%) and an industrial accident in the remaining three (2\%). All fractures were secondary to blunt trauma.

The pelvic radiographs were classified using the current AO/SICOT classification ${ }^{12}$ and the mechanistic classification proposed by Burgess et al ${ }^{13}$ and Dalal et al. ${ }^{14}$ Six sets of radiographs were incomplete or missing and could not be classified; none of these patients had angiography. All patients had rotationally or vertically unstable fractures as defined by the AO/SICOT classification (Table I). There were $94(63 \%)$ rotationally unstable injuries (type B); 62 of these were lateral compression injuries (type B2) making this injury the single commonest subtype. Vertically unstable patterns (type C) were seen in 50 (33\%) patients. Using the Burgess classification ${ }^{13,14}$ there were 21 APC, 75 LC 


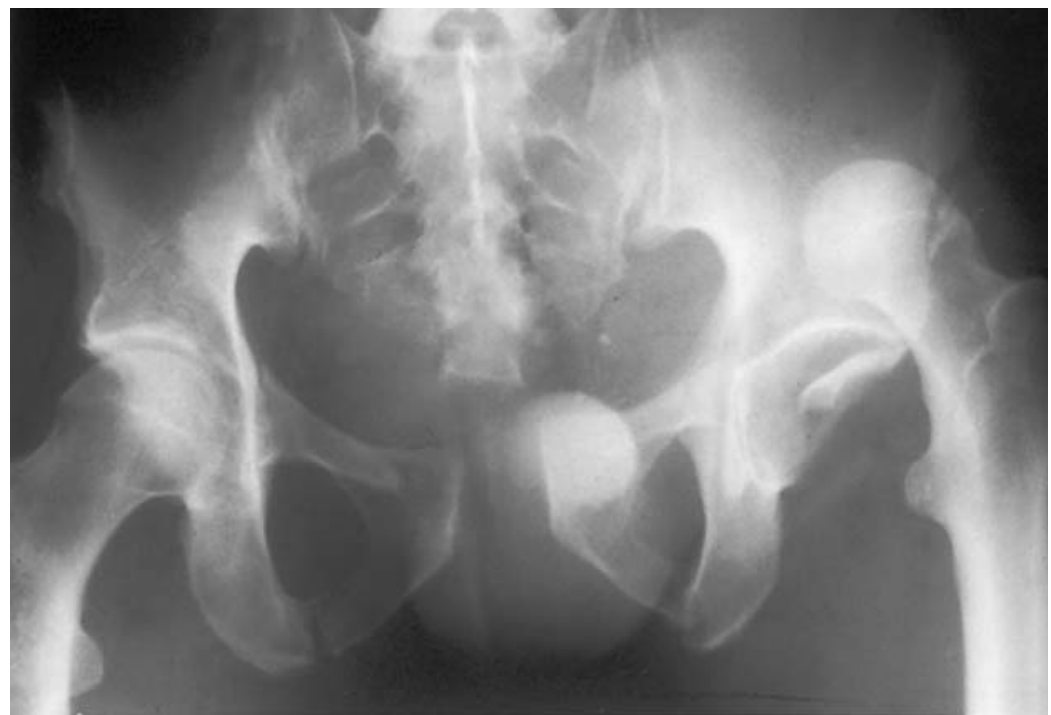

Fig. 1

Radiograph of the pelvis showing disruption of the pelvic ring and dislocation of the left femur.

and 43 VS patterns, with five combined mechanical injuries (Table II).

Of the 150 patients, $74(49 \%)$ had significant hypotension with a systolic blood pressure of less than $90 \mathrm{mmHg}$ at presentation or within 24 hours of admission. Of these 74, 23 had pelvic angiography as part of their emergency management, and this group was studied in detail. There were 19 males and four females with a mean age of 38 years (8 to 74). The mechanism of injury was similar in this subset of patients to that of the whole group; 17 patients were involved in a motor-vehicle accident (eight pedestrians) and six fell from a height. All had severe injuries.

Angiographic technique. Angiography of the pelvic vascular tree was carried out within 24 hours of admission. A Seldinger technique was used to gain access to the femoral artery in the groin. Sites of extravasation of arterial contrast were identified, and selectively cannulated with gelfoam or Gianturco stainless-steel coils (Fig. 2).
Data regarding the sequence and timing of fixation, the relationship to angiography and the need for laparotomy or other operative treatment were recorded prospectively. The response of hypotension to surgical intervention was analysed. The need for transfusion in the first 24 hours after admission was also assessed. The main outcome measures were the control of hypotension in patients requiring embolisation, complications related to the procedure and subsequent mortality.

\section{Results}

The median injury severity score was 20 (9 to 75$)$ in patients who did not have angiography, and 34 (20 to 57) in those who did (Mann-Whitney U test, $\mathrm{p}<0.008$ ). The median injury score of the entire group was 25 (16 to 25). Eight of the 23 patients who had angiography (35\%) had a Glasgow Coma Scale of 8 or less on admission. Five had

Table I. The AO/SICOT classification ${ }^{12}$

\begin{tabular}{lll}
\hline Type & Number of cases (\%) & Angiography \\
\hline B1 (open book) & $18(13)$ & 3 \\
B2 (lateral compression) & $62(43)$ & 7 \\
B3 (combination of above two) & $14(10)$ & 1 \\
C1 (unilateral vertical shear) & $41(28)$ & 8 \\
C2 (vertical shear; contralateral rotational) & $4(3)$ & 0 \\
C3 (bilateral vertical shear) & $5(3)$ & 4 \\
\hline
\end{tabular}

Table II. The Burgess classification ${ }^{13,14}$

\begin{tabular}{llc}
\hline Type & Number of cases (\%) & Angiography \\
\hline Anterior posterior compression (APC) & $21(15)$ & 3 \\
Lateral compression (LC) & $75(52)$ & 8 \\
Vertical shear (VS) & $43(30)$ & 12 \\
Combined mechanical injury & $5(3)$ & 0 \\
\hline
\end{tabular}




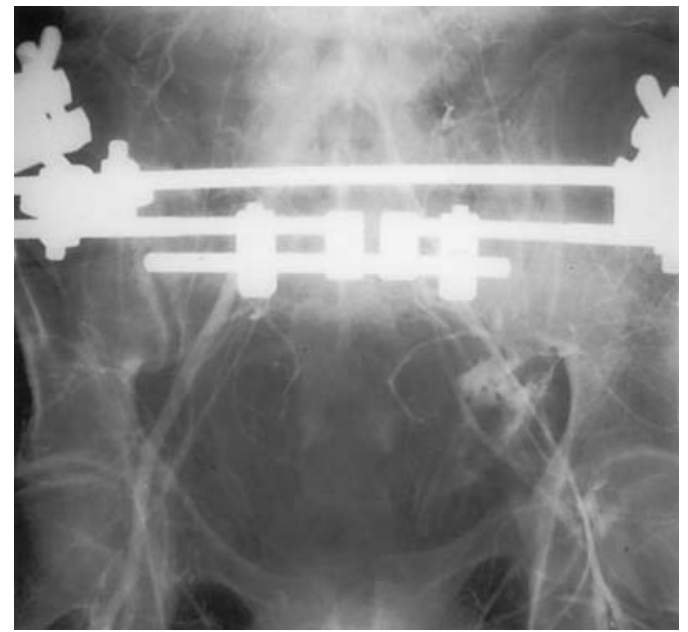

Fig. 2a

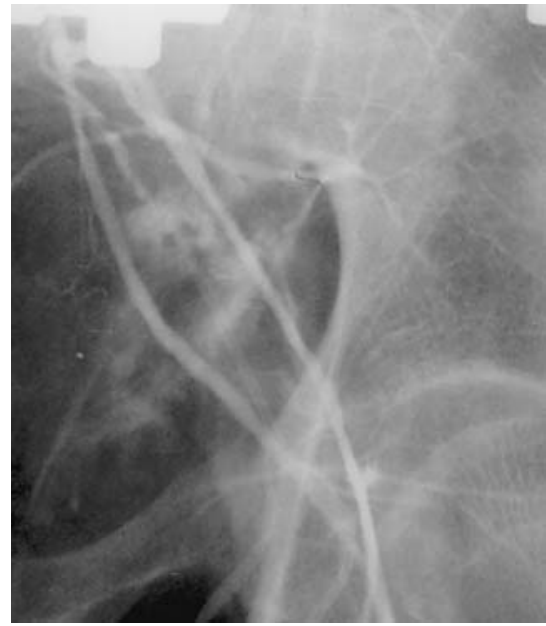

Fig. 2b

Aortogram after application of an external fixator frame showing a) extravasation of dye from the left superior gluteal artery and b) enlarged view of the jet of dye from same vessel before embolisation with incongruent reduction of the femoral head.

significant intrathoracic trauma with an abbreviated injury score of 4 or 5 .

Using the AO/SICOT classification there were 11 rotationally unstable patterns (three type B1, seven type B2 and one type B3) and 12 vertically unstable injuries (eight type $\mathrm{C} 1$ and four type $\mathrm{C} 3$ ). Using the Burgess classification there were three APC, eight LC and 12 VS injuries. Angiography was performed in $14 \%$ of APC, $11 \%$ of LC and $28 \%$ of VS injuries. The requirement for angiography did not change over the period of the study. It was $16 \%$ (14/90) between 1988 and 1993 and 15\% (9/60) in the latter part of the study.

Five fractures were open. Twenty patients were hypotensive on admission and remained so despite intravenous fluid replacement. The remaining three were initially resuscitated but developed further periods of hypotension which were resistant to fluid replacement. Initial investigations included diagnostic peritoneal lavage (DPL) in eight cases, of which four were positive. Fifteen patients had CT of the brain, which was positive for intracranial trauma in ten. The mean time from presentation in the accident and emergency department to angiography was three hours and 45 minutes. The mean duration of the angiographic procedure was 102 minutes (30 to 150 ).

The initial treatment in nine patients was the emergency application of an external fixator (one APC, two LC, six VS). Six of these had a laparotomy to deal with intraperitoneal haemorrhage during the same visit to theatre. The retroperitoneal haematoma was not explored in these cases. Hypotension persisted and angiography was subsequently performed. Thirteen patients underwent angiography as the primary procedure, before any other intervention (two APC, six LC, five VS). Eight of these subsequently had an external fixator frame applied and two had a defunctioning colostomy for fractures communicating with the rectum. One patient had internal fixation of a symphyseal diastasis associated with a VS injury but became hypotensive within 24 hours of surgery and angiography was performed at that stage.

In 18 patients (78\%), sources of extravasation of arterial contrast were identified (two APC, seven LC and nine VS). The remaining five angiograms did not demonstrate this (one APC, one LC and three VS). Embolisation of both internal iliac arteries with gelatine sponge and Gianturco stainless-steel coils was performed in two patients and of the left internal iliac in three. Embolisation of branches of the internal iliac was performed on the right in seven, the left in five and bilaterally in one patient. This was successful in stopping sources of haemorrhage which were identified angiographically in all cases. The morphology of the fracture was not a reliable guide to the associated vascular injury. Complications directly related to the angiography included a unilateral gluteal infarction and a false aneurysm of the external iliac artery.

The mean number of units of blood transfused in the first 24 hours was 25 . Ten of the patients (43\%) died. Two of these remained hypotensive despite embolisation and died in the angiography suite. The remaining eight had their hypotension controlled and were returned to the intensivecare unit; six of these died within 24 hours of admission of whom five had severe head injuries which were the main cause of death. The sixth was an elderly patient with multiple injuries. Two patients died at six and seven days after injury of multiple organ dysfunction syndrome. Three deaths occurred in the LC group and seven in the VS group, a mortality rate of $37 \%$ and $58 \%$, respectively. The mortality rate among patients who did not have angiography was 15\% (19/127 patients). These differences were statistically significant (chi-squared test, $\mathrm{p}<0.003$, Table III). The extent of embolisation required had some predictive value 
Table III. Analysis of outcome

\begin{tabular}{llllc}
\hline & No angiogram & Negative angiogram & Positive angiogram & Total \\
\hline Fatal & 19 & 1 & 9 & 29 \\
Survivor & 108 & 4 & 9 & 121 \\
\hline
\end{tabular}

Table IV. Summary of results of angiography in previous studies

\begin{tabular}{lcccc}
\hline Author/s & Pelvic fractures & Angiography & Embolised & Mortality \\
\hline Gilliland et al $^{10}$ & 100 & 18 (unstable) & 6 & $1 / 6$ \\
Mucha and Fernell $^{17}$ & 533 & 13 & 11 & $5 / 13$ \\
Panetta et al $^{16}$ & 31 & 31 & 27 & $11 / 31$ \\
Moreno et al $^{4}$ & 538 & 7 & 3 & $1 / 3$ \\
Evers et al $^{1}$ & 245 & 16 & 9 & $8 / 9$ \\
Agolini et al & 806 & 35 & 15 & $7 / 15$ \\
Subtotal & 2253 & 120 & 71 & $33 / 77$ \\
Our study & 150 & 23 & 18 & $10 / 23$ \\
Total & 2403 & 143 & 89 & $43 / 100$ \\
\hline
\end{tabular}

regarding outcome. Three of the five patients who had one or both internal iliac arteries embolised died.

\section{Discussion}

Our study indicates that pelvic angiography and embolisation are useful in the management of severe hypotension after major fractures of the pelvis. It also confirms the poor prognosis for these patients, with an overall rate of mortality of $43 \%$. Those patients who required angiography had a much higher injury severity score than those who did not. Mortality was usually due to the associated injuries rather than to uncontrolled hypotension.

A review of the orthopaedic literature gives little information on the use of angiography in patients with pelvic fractures. Previous large series of pelvic fractures do not discuss the role of angiography and embolisation or its relation to the morphology of the fracture. ${ }^{1,4,10,15-17}$ Table IV gives a summary of the results of these papers. The mortality rate of $43 \%$ (33/77) is similar to that in our series. The overall requirement for angiography was 5\% $(120 / 2253)$, which is much smaller than the $15 \%$ in our patients. However, these studies included all types of pelvic fracture irrespective of stability. Our study group included only those patients with rotationally or vertically unstable pelvic fractures as defined by two widely accepted classification systems. These patients are the most likely to develop resistant hypotension. The main drawback of previous studies was the small numbers of patients who were evaluated by angiography. No attempt has been made to relate the incidence of hypotension to currently accepted classifications of fractures of the pelvis. Our study is a detailed account of patients undergoing angiography with an analysis of the factors which influence the outcome.

VS injuries comprised $29 \%$ of fractures in our series; $28 \%$ (12/43) required angiography. They accounted for $52 \%(12 / 23)$ of the group undergoing angiography. This reflects the higher energy associated with this pattern of fracture and the increased risk of serious associated vascular injury and refractory hypotension. By contrast, the need for angiography and embolisation was much lower in patients with APC and LC injuries, only 14\% (3/21) and $11 \%(8 / 75)$ respectively. One other notable finding in our study, however, was that the morphology of the fracture was not predictive of the vascular lesion identified at angiography.

The decision to perform angiography and the most appropriate timing continue to be sources of controversy and represent the most difficult aspects of management. There is often confusion as to whether to perform a laparotomy and the optimum sequence of operative management. Of the ten patients who died in our series, six underwent angiography as the first therapeutic intervention;

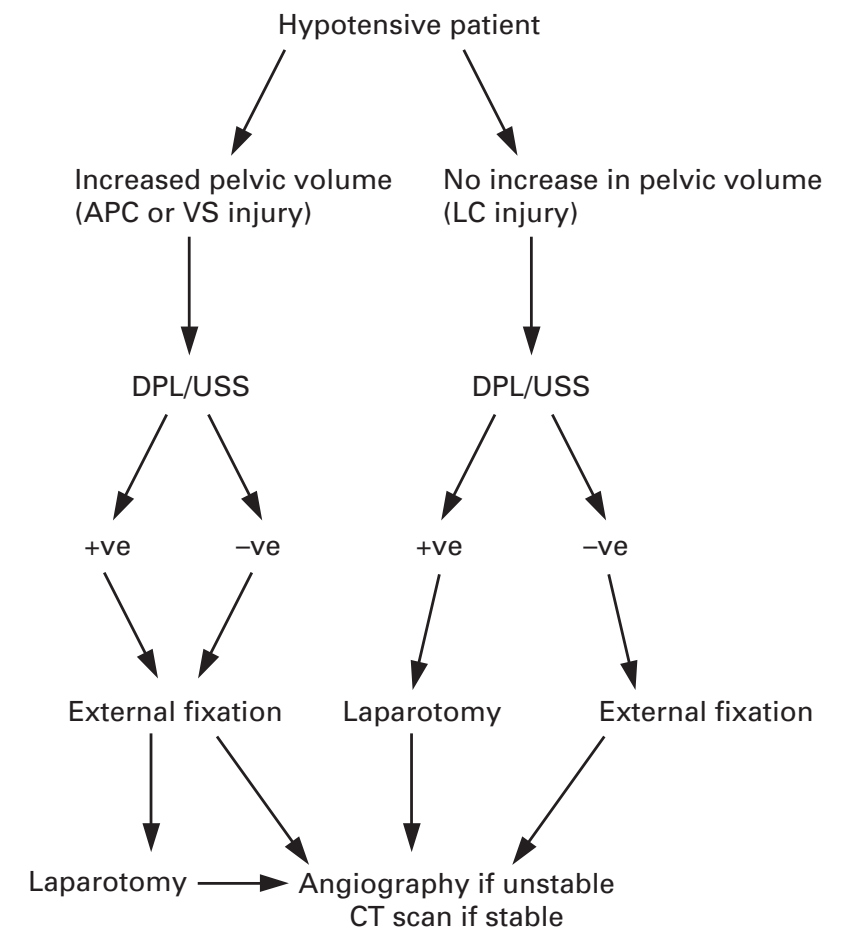

Fig. 3

Algorithm for the management of hypotension in patients with a fracture of the pelvis after inadequate intravenous fluid replacement. 
five of these had a fracture with an increase in pelvic volume (APC or VS injuries) which could have been stabilised by an external fixator. Two of these five died of uncontrolled hypotension in the angiography suite. A reduction in the pelvic volume by the application of an external frame should have been the first step in management.

We have developed an algorithm to guide management (Fig. 3). A strongly positive DPL or increasing volumes of free intraperitoneal fluid on serial ultrasound scans are indications for laparotomy. There is clinical evidence to show that the use of external fixation in fractures associated with an increase in pelvic volume helps to obtain haemodynamic stability. ${ }^{3,6,17-19}$ Accordingly, we consider that this should be the first procedure after fluid resuscitation in patients with an increased pelvic volume. If the fixator is not applied before laparotomy the increase in the retroperitoneal haematoma which occurs when the abdomen is open may make closure of the wound difficult or impossible.

Those patients who do not have an increase in pelvic volume (i.e., LC injuries) should have a laparotomy to treat intraperitoneal sources of blood loss if indicated by the results of the DPL or ultrasound. The advantage of an external fixator is uncertain in these patients but it may enhance stability and can be applied relatively quickly if there is delay in obtaining angiography. Patients in either group who have persistent hypotension despite these measures should be considered for angiography.

There are a number of drawbacks to angiography. It is a time-consuming procedure and involves the transportation of a severely injured patient to an angiography suite. It requires the availability of a skilled interventional radiologist. Anatomical studies ${ }^{11}$ suggest that the bony surfaces of the fracture and veins are the main sources of haemorrhage. On this basis some feel that arteriography is rarely required. ${ }^{20}$ This view is not borne out by our study or other reports. $^{1,4,10,15-17}$ Significant sources of arterial haemorrhage were identified in $78 \%$ of our cases and in over $59 \%$ (71/120) of patients in other studies. Embolisation of these vessels in our patients facilitated control of the hypotension.

The alternative to pelvic angiography is immediate exploration of the pelvic retroperitoneum to achieve control of arterial blood loss and packing to control venous bleeding. The packs are changed or removed at a second procedure, usually 48 hours later. Although this approach has been practised in some level-1 trauma centres in Europe, ${ }^{9}$ there are few published data to support its use. It is likely to be indicated mainly for a small group of patients with severe refractory hypotension who have an imminent prospect of death and will not survive the transfer for angiography.

The high mortality encountered in our study is a reflection of the severity of the pelvic injury and the presence of concomitant injuries, in particular cranial trauma. The patients who required angiography had a higher injury severity score. The mortality in most of our patients was not due to uncontrolled hypotension but to associated injuries, in particular severe head injury.

We conclude that pelvic angiography should be reserved for patients in whom hypotension is unresponsive to other interventions. In these difficult cases arterial sources of blood loss are demonstrable in most patients and embolisation can help to control hypotension. Although the mortality is high, the results of our study support the view that angiography and embolisation are worthwhile procedures.

No benefits in any form have been received or will be received from a commercial party related directly or indirectly to the subject of this article.

\section{References}

1. Evers BM, Cryer HM, Miller FB. Pelvic fracture haemorrhage: priorities in management. Arch Surg 1989;124:422-4.

2. Ghanayem AJ, Stover MD, Goldstein JA, Bellon E, Wilber JH. Emergent treatment of pelvic fractures: comparison of methods for stabilization. Clin Orthop 1995;318:75-80.

3. Klein SR, Saroyan RM, Baumgartner F, Bongard FS. Management strategy of vascular injuries associated with pelvic fractures. J Cardiovasc Surg (Torino) 1992;33:349-57.

4. Moreno C, Moore EE, Rosenberger A, Cleveland HC. Haemorrhage associated with major pelvic fracture: a multispecialty challenge. J Trauma 1986;26:987-94.

5. Rothenberger DA, Fischer RP, Strate RG, Velasco R, Perry JF Jr. The mortality associated with pelvic fractures. Surgery 1978;84:356-61.

6. Mears DC, Fu FH. Modern concepts of external skeletal fixation of the pelvis. Clin Orthop 1980;151:65-72.

7. Batalden DJ, Wickstrom PH, Ruiz E, Gustilo MD. Value of the G suit in patients with severe pelvic fracture: controlling hemorrhagic shock. Arch Surg 1974;109:326-8.

8. Riska EB, von Bonsdorff H, Hakkinen S, et al. Operative control of massive haemorrhage in comminuted pelvic fractures. Int Orthop 1979;3:141-4.

9. Pohlemann T, Bosch U, Gansslen A, Tscherne H. The Hannover experience in management of pelvic fractures. Clin Orthop 1994;305:69-80.

10. Gilliland MG, Ward RE, Flynn TC, et al. Peritoneal lavage and angiography in the management of patients with pelvic fractures. Am J Surg 1982;144:744-7.

11. Huittinen VM, Slatis P. Postmortem angiography and dissection of the hypogastric artery in pelvic fractures. Surgery 1973;73:454-62.

12. Tile M. Classification of fractures of the pelvis and acetabulum. In: Tile M, ed. Fractures of the pelvis and acetabulum. 2nd edition. London, etc: Williams \& Wilkins, 1995:66-101.

13. Burgess AR, Eastridge BJ, Young JWR, et al. Pelvic ring disruptions: effective classification system and treatment protocols. J Trauma 1990;30:848-56.

14. Dalal SA, Burgess AR, Siegel JH, et al. Pelvic fracture in multiple trauma: classification by mechanism is key to pattern of organ injury, resuscitative requirements and outcome. $J$ Trauma 1989;29:981-1000.

15. Agolini SF, Shah K, Jaffe J, et al. Arterial embolisation is a rapid and effective technique for controlling pelvic fracture haemorrhage. $J$ Trauma 1997;43:395-9.

16. Panetta T, Sclafani SJ, Goldstein AS, Phillips TF, Shaftan GW. Percutaneous transcatheter embolization for massive bleeding from pelvic fractures. J Trauma 1985;25:1021-9.

17. Mucha P, Farnell MB. Analysis of pelvic fracture management. $J$ Trauma 1984;24:379-86.

18. Gylling SF, Ward RE, Holcroft JW, et al. Immediate external fixation of unstable pelvic fractures. Am J Surg 1985;150:721-4.

19. Routt ML Jr, Simonian PT, Ballmer F. A rational approach to pelvic trauma: resuscitation and early definitive stabilisation. Clin Orthop 1995;318:61-74.

20. Tscherne H, Regel G. Care of the polytraumatised patient. $J$ Bone Joint Surg [Br] 1996;78-B:840-52. 